\title{
Treat the patient by the recognized toxidrome when the ingested herbal juice is non-toxic
}

\author{
Yu-Jang Su • Yen-Chun Lai
}

Received: 27 September 2009 / Accepted: 25 February 2010/Published online: 12 May 2010

(C) Springer-Verlag London Ltd 2010

\begin{abstract}
This is the case of 63-year-old mother and her 35-year-old daughter who drank herbal juice (Rhizoma Dioscoreae Nipponicae and Ficus formosana Maxim) and then developed diarrhea, cold sweating, and myoclonus. On physical examination, the mother had a normal consciousness level, a normal respiratory rate, and bilateral miosis. After the administration of $2 \mathrm{~g}$ of pralidoxime (PAM) and $0.5 \mathrm{mg}$ of atropine, the mother felt better. The daughter only had mild symptoms of sweating and vomiting before arrival at the emergency department (ED).

The poison center of our hospital was consulted, and they assured us that the herbs ingested were all non-toxic. In conclusion, the majority of emergency physicians are not familiar with herbal medicines and plants. In this instance, however, it was more important to apply appropriate early management according to the toxidrome rather than to recognize what kind of herb had been ingested.
\end{abstract}

\section{Y.-J. Su $(\bowtie)$}

Department of Emergency Medicine, Mackay Memorial Hospital, No. 92, Sec. 2, North Chung-Shan Rd.,

10449 Taipei, Taiwan

e-mail: yjsu@ms1.mmh.org.tw

\section{Y.-J. Su}

Mackay Medicine, Nursing and Management College,

No. 92, Shengjing Rd., Beitou District,

Taipei 112, Taiwan

\section{Y.-J. Su}

Research Center for Biomedical Implants and Microsurgery

Devices, Taipei Medical University,

110 Taipei, Taiwan

Y.-C. Lai

Department of Anesthesiology, Wan Fang Hospital,

Taipei Medical University,

110 Taipei, Taiwan
Keywords Herbal medicine - Rhizoma Dioscoreae Nipponicae $\cdot$ Ficus formosana Maxim · Toxidrome

A 63-year-old mother and her 35-year-old daughter presented to our emergency department (ED) with diarrhea, cold sweating, and muscle tremors. They had ingested $150 \mathrm{cc}$ of the cooked herbal drugs Rhizoma Dioscoreae Nipponicae and Ficus formosana Maxim $1 \mathrm{~h}$ prior to arrival at the ED (Fig. 1). The daughter vomited once, and expelled most of the herbal juice that she had drunk, so she exhibited only mild sweating after arrival to the ED. The mother had severe muscle tremors, sweating, watery diarrhea, and bilateral small pupil size of $1.5 \mathrm{~mm}$. Her vital signs were: body temperature, $36{ }^{\circ} \mathrm{C}$; pulse rate, 66 beats/ minute; respiratory rate, 20 breaths/minute; blood pressure, $89 / 44 \mathrm{mmHg}$. No neurological deficits were found in either the mother or the daughter. We consulted the poison center of our hospital and knew the herbs were all non-toxic. After the administration of $2 \mathrm{~g}$ of pralidoxime (PAM) and $0.5 \mathrm{mg}$ of atropine, the mother felt better. They recovered and were discharged $7 \frac{1}{2} \mathrm{~h}$ after close observation in the emergency department.

Usage of cholinergic agents, opiates/opioids, and sedative hypnotics, as well as pontine hemorrhage are all in the list of differential diagnoses for the causes of miosis [1]. According to the clinical history, the patients had not taken hypnotics prior to arrival at the hospital, and there was no evidence of focal neurological signs or low respiratory rate. The mother had five episodes of watery diarrhea, persistent myoclonus, and hypotension, and a cholinergic toxidrome was highly suspected to be present in this mother and daughter. Nasogastric tube insertion and irrigation were applied, and the patients were also given $50 \mathrm{~g}$ of activated charcoal for gastrointestinal (GI) decontamination. After the administration of $2 \mathrm{~g}$ of pralidoxime (PAM) and $0.5 \mathrm{mg}$ of atropine, the mother felt better. 
Fig. 1 The cooked herbal medicine Rhizoma Dioscoreae Nipponicae and Ficus formosana Maxim, which have the effect of strengthening the musculo-skeletal system

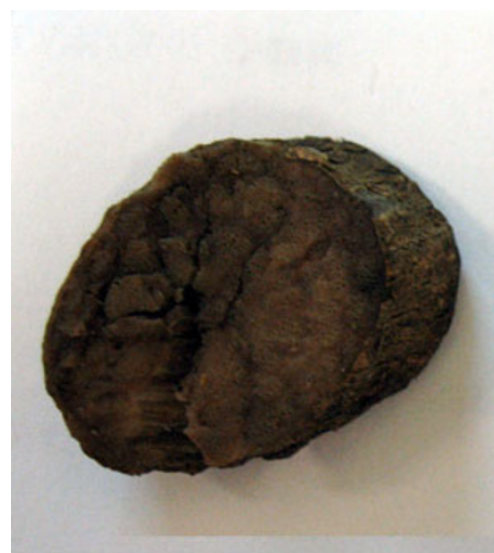

Ficus formosana Maxim.

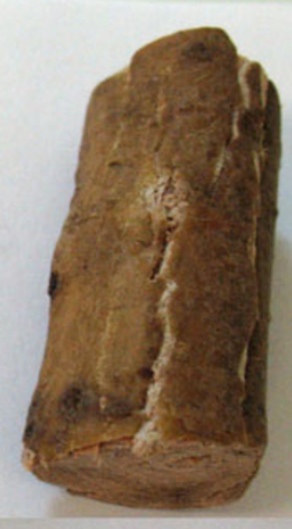

Rhizoma Dioscoreae Nipponicae
The patients recovered and were discharged $71 / 2 \mathrm{~h}$ after close observation in the emergency department.

A toxidrome is a syndrome caused by the presence of a hazardous level of toxins in the body. It can be recognized by many clinical features, such as vital signs, consciousness level, size of the pupils, volume of secretions, motility of gastrointestinal tract, urination, and dry/wet skin. In our case, the small pupillary sizes, myoclonus, and frequent diarrhea pointed to a cholinergic toxidrome.

Most emergency physicians probably are not familiar with all herbal medicines and plants. But this case highlights the importance of applying appropriate management immediately according to the presumed toxidrome rather than recognition of what kind of herb was ingested [2].

\section{References}

1. Challoner KR, McCarron MM, Newton EJ (1990) Pentazocine (Talwin) intoxication: report of 57 cases. J Emerg Med 8(1):67-74

2. Lin TJ, Nelson LS, Tsai JL et al (2009) Common toxidromes of plant poisonings in Taiwan. Clin Toxicol (Phila) 47(2):161-168 